\title{
Prognostic significance of miR-218 in human hepatocellular carcinoma and its role in cell growth
}

\author{
KANGSHENG TU, CHAO LI, XIN ZHENG, WEI YANG, YINGMIN YAO and QINGGUANG LIU \\ Department of Hepatobiliary Surgery, The First Affiliated Hospital of Xi'an \\ Jiaotong University, Xi'an, Shaanxi 710061, P.R. China
}

Received April 5, 2014; Accepted June 16, 2014

DOI: $10.3892 /$ or.2014.3386

\begin{abstract}
MicroRNA-218 (miR-218) is considered a tumor suppressor in human cancer. In the present study, miR-218 expression was found to be significantly lower in human hepatocellular carcinoma (HCC) than in normal tumor-adjacent tissues. miR-218 was clearly silenced or downregulated in five HCC cells (HepG2, Hep3B, SMMC-7721, Huh7 and Bel-7402) compared with normal hepatocytes (LO2). The low expression of miR-218 conferred a poor 5-year survival in HCC patients. Multivariate Cox regression analysis indicated that miR-218 was an independent prognostic factor in HCC. Ectopic expression of miR-218 inhibited proliferation and promoted apoptosis in HepG2 and SMMC-7721 cells. In tumor bearing mice, miR-218 slowed down tumor growth by inducing apoptosis and growth arrest. Restoring miR-218 expression resulted in downregulation of B lymphoma Mo-MLV insertion region 1 homolog (BMI-1) mRNA and protein level in HepG2 and SMMC-7721 cells. In addition, BMI-1 mRNA expression in HCC was significantly higher than that in non-cancerous tissues. BMI-1 mRNA was inversely correlated with miR-218 expression in HCC tissues. In conclusion, miR-218 may serve as a prognostic biomarker and induce apoptosis and growth arrest by downregulating BMI-1 in HCC.
\end{abstract}

\section{Introduction}

Hepatocellular carcinoma (HCC) is one of the most common malignancies and the second leading cause of cancer-related mortality in China (1). Progression of multimodality therapy has improved the outcome for HCC patients, but it has not yet achieved satisfactory curative effect. Therefore, it is important to elucidate the precise molecular mechanisms of HCC development and to develop new therapeutic targets (2).

Correspondence to: Professor Qingguang Liu or Dr Kangsheng Tu, Department of Hepatobiliary Surgery, The First Affiliated Hospital of Xi'an Jiaotong University, Xi'an, Shaanxi 710061, P.R. China

E-mail: liuqingguang@vip.sina.com

E-mail: tks0912@foxmail.com

Key words: miR-218, hepatocellular carcinoma, BMI-1, prognosis, tumor progression
MicroRNAs (miRNAs) are a large class of evolutionarily conserved non-coding RNAs 18-25 nucleotides in length that negatively regulate genes involved in many fundamental cell processes including development, differentiation, proliferation, survival and death (3). Recent studies have shown that miRNAs play key roles in the initiation and progression of cancer (4). Deregulation of miRNAs has been reported in various types of human cancers including lymphoma, colorectal and breast cancer, glioblastoma, lung cancer, papillary thyroid carcinoma and HCC, suggesting it is a hallmark of cancer (5). Specific miRNAs have been shown to regulate known oncogenes or tumor suppressor genes or function as so called onco-miRs or tumor suppressor-miRs by directly targeting other genes involved in cell differentiation, proliferation, invasion, apoptosis and angiogenesis in various types of cancer (4).

miRNAs have been reported to play a critical role in the hepatocarcinogenesis with dysfunction of targeting genes (6). Several miRNAs have been found to be aberrantly expressed in HCC and most of them are related to the malignant behavior of the tumors (7). Recently, microRNA-218 (miR-218) was recognized as a tumor suppressor and its downregulation was found in human cancer, including cervical, gastric, lung, colon, prostate and bladder cancer (8-16). miR-218 suppresses cell proliferation, inhibits cell cycle progression and induces apoptosis in colon cancer by downregulating B lymphoma Mo-MLV insertion region 1 homolog (BMI-1)(13). However, the status, clinical significance and function of miR-218 in HCC remain poorly understood.

In the present study, we demonstrated that reduced miR-218 expression is correlated with poor clinicopathological parameters in HCC. miR-218 is an independent prognostic factor for predicting both the overall and the disease-free 5-year survival of HCC patients. miR-218 functions as a tumor suppressor in HCC by inhibiting cell proliferation and inducing apoptosis in vitro and in vivo. Furthermore, miR-218 negatively regulates BMI-1 abundance in HCC cells and it is inversely correlated with BMI-1 mRNA in HCC tissues. Our results suggest that miR-218 may inhibit BMI-1 expression, thereby inhibiting HCC growth and, hence, tumor progression.

\section{Materials and methods}

Clinical samples and cell lines. This study included a total of $60 \mathrm{HCC}$ patients, including 49 males and 11 females (range, 
36-73 years; median 51 years), who underwent curative liver resection at the Department of Hepatobiliary Surgery, The First Affiliated Hospital Xi'an Jiaotong University from March 2006 to November 2008, with a median follow-up time of 31.5 months. None of the patients received chemotherapy, radiotherapy or radiofrequency ablation before operation. The clinicopathological data are shown in Table I. HCC tissues and matched normal tumor-adjacent tissues $(>2 \mathrm{~cm}$ distance of the surgical margin) were collected and used after obtaining informed consent. The Xi'an Jiaotong University Ethics Committee approved all protocols according to the 1975 Helsinki Declaration.

The human immortalized normal hepatocyte cell line (LO2) and five HCC cell lines (HepG2, Hep3B, SMMC-7721, Bel-7402 and Huh7) were obtained from the Institute of Biochemistry and Cell Biology, Chinese Academy of Sciences (Shanghai, China). Cells were cultured in complete Dulbecco's modified Eagle's medium (DMEM) containing 10\% fetal bovine serum (FBS) (both from Gibco, USA) with $100 \mathrm{U} / \mathrm{ml}$ penicillin and $100 \mu \mathrm{g} / \mathrm{ml}$ streptomycin (Sigma, USA) and cultured in a humidified $5 \% \mathrm{CO}_{2}$ incubator at $37^{\circ} \mathrm{C}$.

Real-time quantitative reverse transcription-PCR ( $q R T-P C R)$. The PCR amplification for the quantification of the miR-218 and RNU6B was performed using TaqMan miRNA Reverse Transcription kit and TaqMan Human MiRNA Assay kit (both from Applied Biosystems, USA). The relative expression of miR-218 was shown as fold difference relative to RNU6B.

BMI-1 sense primers, 5'-GTGCTTTGTGGAGGGTACTT CAT-3' and antisense, 5'-TTGGACATCACAAATAGGACAA TACTT-3'. Total RNA was isolated from HCC tissues and cells using TRIzol ${ }^{\circledR}$ reagent (Invitrogen, USA) according to the manufacturer's protocol. The first strand cDNA was synthesized using the RevertAid ${ }^{\mathrm{TM}}$ First Strand cDNA synthesis kit (Fermentas, USA). cDNA ( $2 \mu \mathrm{l})$ obtained from each sample was amplified and quantified by real-time PCR using SYBR ${ }^{\circledR}$ Premix Ex Taq ${ }^{\mathrm{TM}}$ II (Tli RNaseH Plus; Takara, Japan). The human GAPDH gene served as an internal control gene to ensure that an equal amount of mRNA was analyzed from each sample.

miRNA transfection. Cells were seeded in a $24-$ well plate at the concentration of $1 \times 10^{5} /$ well and divided into two groups (miR-control and miR-218 group). Cells were transfected with pre-miR-218 or pre-miR control at $30 \mathrm{nmol} / \mathrm{l}$ using Lipofectamine 2000 (Invitrogen, USA) according to the manufacturer's guidelines.

Cell proliferation and apoptosis assay. For the proliferation assay, HCC cells were seeded into 96-well plates at 5,000 cells/well for $24 \mathrm{~h}$ and assessed using a Cell Proliferation ELISA, BrdU (chemiluminescent) (Roche, USA), as described in our previous study (17). An Annexin V-FLUOS Staining kit (Roche) was used to analyze the level of apoptosis, as previously described (2).

Western blotting. The following primary antibodies were used in the immunoblotting assays: BMI-1 (D20B7, \#6964; Cell Signaling Technology, USA) (1:1,000) and GAPDH (G8140; US Biological, USA) $(1: 5,000)$. Horseradish peroxidase-conju-
Table I. Clinical significance of miR-218 expression in HCC $(\mathrm{n}=60)$.

\begin{tabular}{|c|c|c|}
\hline $\begin{array}{l}\text { Clinicopathological } \\
\text { characteristics }\end{array}$ & R-value & $\mathrm{P}$-value \\
\hline $\begin{array}{l}\text { Age (years) } \\
<50 \\
\geq 50\end{array}$ & 0.035 & 0.809 \\
\hline $\begin{array}{l}\text { Gender } \\
\text { Male } \\
\text { Female }\end{array}$ & 0.108 & 0.501 \\
\hline $\begin{array}{l}\text { HBV } \\
\text { Absent } \\
\text { Present }\end{array}$ & 0.183 & 0.204 \\
\hline $\begin{array}{l}\text { Serum AFP level (ng/ml) } \\
<400 \\
\geq 400\end{array}$ & -0.159 & 0.221 \\
\hline $\begin{array}{l}\text { Tumor size }(\mathrm{cm}) \\
<5 \\
\geq 5\end{array}$ & -0.429 & $0.029^{\mathrm{a}}$ \\
\hline $\begin{array}{l}\text { No. of tumor nodules } \\
1 \\
\geq 2\end{array}$ & -0.191 & 0.471 \\
\hline $\begin{array}{l}\text { Cirrhosis } \\
\text { Absent } \\
\text { Present }\end{array}$ & -0.203 & 0.352 \\
\hline $\begin{array}{l}\text { Venous infiltration } \\
\text { Absent } \\
\text { Present }\end{array}$ & -0.198 & 0.205 \\
\hline $\begin{array}{l}\text { Edmondson-Steiner grading } \\
\text { I+II } \\
\text { III+IV }\end{array}$ & -0.514 & $0.008^{a}$ \\
\hline $\begin{array}{l}\text { TNM tumor stage } \\
\text { I+II } \\
\text { III+IV }\end{array}$ & -0.571 & $0.002^{\mathrm{a}}$ \\
\hline
\end{tabular}

HCC, hepatocellular carcinoma; HBV, hepatitis B virus; AFP,

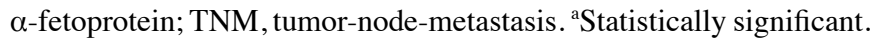

gated goat anti-mouse or anti-rabbit secondary antibodies (Bio-Rad, USA) were used at a 1:1,000-1:5,000 dilution and detected using a western blotting luminol reagent (sc-2048; Santa Cruz, USA), as described in our previous study (2).

In vivo experiments. Female BALB/c nude mice 4-6 weeks old (Centre of Laboratory Animals, The Medical College of Xi'an Jiaotong University, Xi'an, China) were used to establish a nude mouse xenograft model. Mice were housed in sterilized cages ( 2 animals/cage) at a constant temperature and humidity and fed a regular autoclaved chow diet with water ad libitum (2). SMMC- 7721 cells $\left(5 \times 10^{6}\right)$ were inoculated subcutaneously into the flank of each nude mouse. At day 5 after implantation, miR-control or miR-218 was injected into 

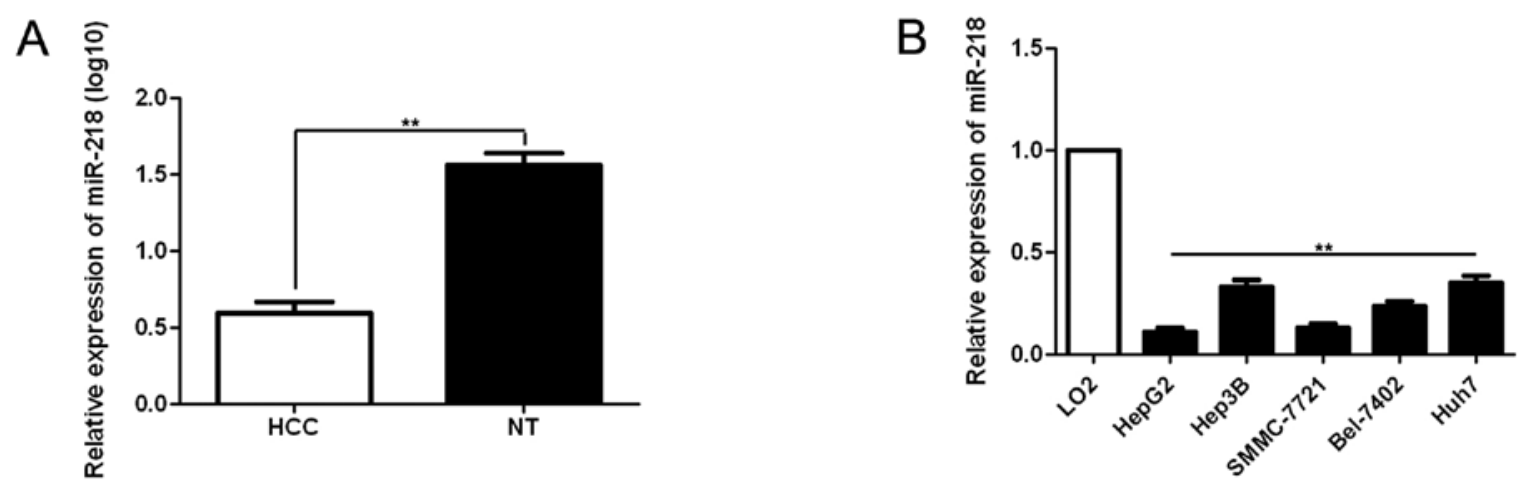

Figure 1. Expression of miR-218 in HCC tissue samples and cells. (A) The expression of miR-218 in cancer (HCC) and non-cancerous tissues (NT) from HCC patients by qRT-PCR. $n=60$, values are depicted as means \pm SEM; ${ }^{* *} \mathrm{p}<0.01$ by t-test. (B) The expression of miR-218 in normal hepatocytes (LO2) and five HCC cells (HepG2, Hep3B, SMMC-7721, Bel-7402 and Huh7). $\mathrm{n}=6$, values are depicted as means \pm SEM; ${ }^{* *} \mathrm{p}<0.01$ by t-test.

the tumor every 3 days, respectively ( $n=6$ mice each group). miRNA (1.2 nmol) was mixed with $10 \mu 1$ Lipofectamine 2000 and incubated for $15 \mathrm{~min}$, then injections were made in a final volume of $100 \mu \mathrm{l}$ in McCoy's 5A medium (Sigma-Aldrich, USA) (13). The tumor volume for each mouse was determined by measuring two of its dimensions and then calculated as Tumor volume $=$ length $\mathrm{x}$ width $\mathrm{x}$ width $/ 2$. After 3 weeks, the mice were sacrificed by cervical dislocation under anesthesia with ether and the xenograft tumor tissue was explanted for routine pathological examination. The amount of apoptosis in the isolated tumor tissues was detected using a TUNEL assay kit (4810-30-K; R\&D Systems, USA) according to the manufacturer's guidelines. All animal protocols were approved by the Institutional Animal Care and Use Committee of Xi'an Jiaotong University.

Immunohistochemical staining. Immunohistochemistry was performed on paraformaldehyde-fixed paraffin sections. Ki-67 (D2H10, \#9027; Cell Signaling Technology) (1:400) antibodies were used in immunohistochemistry with the streptavidin peroxidase-conjugated (SP-IHC) method. Immunohistochemistry was performed as previously reported (18).

Statistical analysis. All data are presented as the means \pm SEM. The SPSS statistical package for Windows version 13 (SPSS, USA) was used for the multi-variant Cox regression analysis. A two-tailed Student's t-test, a Spearman's rank correlation coefficient test, a Kaplan-Meier plot, a log-rank test or an ANOVA was used to evaluate statistical significance using GraphPad Prism 5 software (GraphPad Software, Inc., USA). p $<0.05$ was considered to indicate a statistically significant difference.

\section{Results}

Clinical significance of reduced miR-218 expression in HCC specimens. Previous studies reported that miR-218 expression is impaired in various types of human cancers (8-16). To determine the status of miR-218 and its clinical significance in $\mathrm{HCC}$, we tested miR-218 expression by qRT-PCR in a retrospective cohort of 60 pairs of HCC and matched normal tumor-adjacent tissues from HCC patients who received liver resection. In these cases, we found that miR-218 expression in HCC was significantly lower than that in matched non-cancerous tissues (the mean of $\log 10$ was 0.59 in the tumors and 1.56 in the matched non-tumor tissues, $\mathrm{p}<0.01$, Fig. 1A). Of these 60 paired samples, $75.00 \%(45 / 60)$ of the HCC tissues showed lower miR-218 expression as compared with matched normal tumor-adjacent tissues. Furthermore, we detected miR-218 expression in normal hepatocyte cell line (LO2) and five HCC cell lines (Hep3B, HepG2, SMMC-7721, Huh7 and Bel-7402). Consistent with the tissue samples, miR-218 expression was downregulated in all HCC cell lines as compared with that in the normal hepatocyte cell line $(\mathrm{p}<0.01$, Fig. $1 \mathrm{~B})$. As shown in Table I, clinical significance analysis using a Spearman's rank correlation coefficient test indicated that the miR-218 expression in HCC tissues was significantly associated with a large tumor size $(\mathrm{r}=-0.429, \mathrm{p}=0.029)$, high Edmondson-Steiner grading $(\mathrm{r}=-0.514, \mathrm{p}=0.008)$ and advanced TNM tumor stage $(\mathrm{r}=-0.571, \mathrm{p}=0.002)$.

Reduced miR-218 expression correlates with a poorer 5-year survival for HCC patients. To determine the prognostic significance of miR-218 in HCC patients, quantification of miR-218 was performed to confirm the correlation between miR-218 expression and 5-year patient survival. We constructed KaplanMeier survival curves using the overall 5-year patient survival date to analyze cases with high and low miR-218 expression. Our data suggested overall survival in the miR-218 high expression group was $46.67 \%$, compared with $17.78 \%$ in the low expression group. According to the overall survival curve, patients in the miR-218 low expression group $(n=45)$ had a significantly poorer prognosis than those in the miR-218 high expression group ( $n=15$; log-rank=5.037; $\mathrm{p}=0.0248$, Fig. $2 \mathrm{~A}$ ). The median disease-free survival times in the miR-218 high and low expression subgroups of HCC patients were 38.0 and 20.0 months, respectively. Kaplan-Meier analysis also revealed that miR-218 loss was associated with a shorter disease-free survival time $(\log$-rank=4.163; $\mathrm{p}=0.0413$, Fig. 2B). These data indicate that miR-218 may act as a potential biomarker for predicting prognosis in HCC. Furthermore, multivariate Cox regression analysis indicated that miR-218 expression was an independent factor for predicting both 5-year overall and disease-free survival in HCC patients ( $\mathrm{p}=0.003$ and 0.011 , respectively, Table II). 
Table II. Multivariate Cox regression analysis of 5-year overall and disease-free survival of 60 HCC patients.

\begin{tabular}{|c|c|c|c|c|c|c|}
\hline \multirow[b]{2}{*}{ Variables } & \multicolumn{3}{|c|}{ Overall survival } & \multicolumn{3}{|c|}{ Disease-free survival } \\
\hline & HR & $95 \% \mathrm{CI}$ & $\mathrm{P}$-value & HR & $95 \% \mathrm{CI}$ & P-value \\
\hline Age (years) & 1.365 & $0.559-3.333$ & 0.494 & 0.978 & $0.465-2.057$ & 0.954 \\
\hline Gender & 0.673 & $0.309-1.466$ & 0.319 & 0.916 & $0.442-1.896$ & 0.813 \\
\hline HBV & 2.027 & $0.789-5.205$ & 0.142 & 2.219 & $0.937-5.258$ & 0.070 \\
\hline No. of tumor nodules & 0.766 & $0.368-1.595$ & 0.476 & 0.686 & $0.343-1.370$ & 0.285 \\
\hline Tumor size & 1.655 & $0.731-3.745$ & 0.227 & 1.468 & $0.691-3.120$ & 0.318 \\
\hline Venous infiltration & 1.558 & $0.738-3.291$ & 0.245 & 1.828 & $0.903-3.700$ & 0.094 \\
\hline Serum AFP level & 0.951 & $0.446-2.025$ & 0.896 & 1.019 & $0.514-2.020$ & 0.956 \\
\hline Cirrhosis & 1.634 & $0.834-3.204$ & 0.468 & 1.561 & $0.859-2.838$ & 0.144 \\
\hline Edmondson-Steiner grading & 0.636 & $0.280-1.443$ & 0.279 & 0.600 & $0.295-1.218$ & 0.157 \\
\hline TNM tumor stage & 0.208 & $0.074-0.585$ & $0.003^{\mathrm{a}}$ & 0.297 & $0.124-0.710$ & $0.006^{\mathrm{a}}$ \\
\hline miR-218 expression in tumor & 3.475 & $1.515-7.972$ & $0.003^{\mathrm{a}}$ & 2.547 & $1.240-5.232$ & $0.011^{\mathrm{a}}$ \\
\hline
\end{tabular}

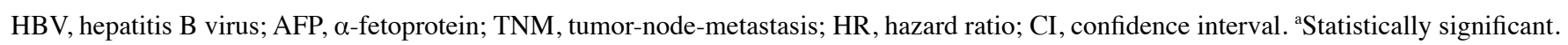
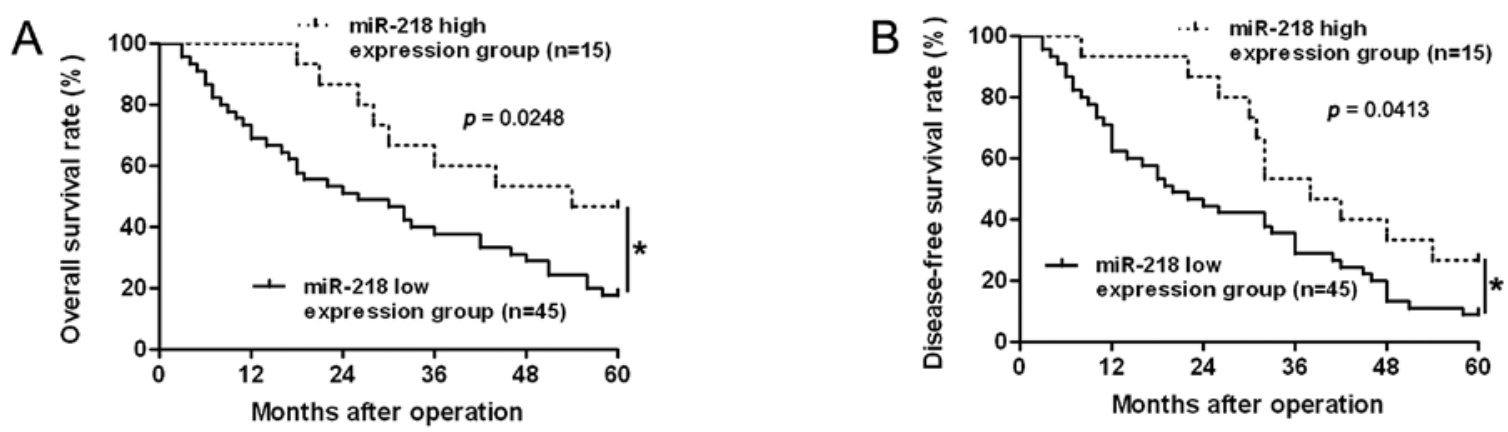

Figure 2. Clinical significance of miR-218 in HCC cases. (A) Kaplan-Meier 5-year overall survival curves of HCC patients according to the level of miR-218 expression. (B) Kaplan-Meier 5-year disease-free survival curves of HCC patients according to the level of miR-218 expression. The miR-218 high expression group ( $\mathrm{n}=15)$, miR-218 (HCC)/miR-218 (NT) $\geq 1.0$; miR-218 low expression group ( $\mathrm{n}=45)$, miR-218 (HCC)/miR-218 (NT) $<1.0$; ${ }^{*} \mathrm{p}<0.05$ by log-rank test.

miR-218 inhibits proliferation and promotes apoptosis in HCC cells. Previous studies demonstrated that miR-218 acts as a tumor suppressor by inducing apoptosis and growth arrest (13). To identify the role of miR-218 in HCC, we restored miR-218 expression in two HCC cell lines, HepG2 and SMMC-7721. As assessed by qRT-PCR, the miR-218 level was raised by ectopically expressing pre-miR-218 in both cell lines ( $p<0.01$, respectively, Fig. 3A). BrdU assays were performed to test the effect of altering miR-218 levels on tumor cell proliferation. We found that miR-218 overexpression led to a significant reduction of cell proliferation in both HepG2 and SMMC-7721 cells ( $\mathrm{p}<0.01$, respectively, Fig. 3B). Furthermore, as determined by flow cytometry, the percentage of apoptotic HepG2 and SMMC-7721 cells was significantly elevated after miR-218 overexpression ( $\mathrm{p}<0.01$, respectively, Fig. $3 \mathrm{C}$ ). Thus, miR-218 may exert an anti-HCC effect by promoting both apoptosis and growth arrest.

miR-218 inhibits tumor growth in mice. We next sought to determine whether miR-218 affects tumor growth using an SMMC-7721 subcutaneous tumor model. Mice were treated with miR-218 or miR-control by multi-center intratumoral injection. Tumor growth curves revealed that miR-218 slowed down tumor growth in mice ( $p<0.01$, Fig. 4A). Furthermore, we performed immunohistochemistry for Ki-67 and TUNEL assays in the xenografted tissues. Consistent with our in vitro data, miR-218 inhibited proliferation and induced apoptosis in vivo $(\mathrm{p}<0.01$, respectively, Fig. $4 \mathrm{~B})$.

miR-218 regulates BMI-1 abundance in HCC cells. Previous studies reported that BMI-1 was a potential oncogene in human cancers and it was suppressed by miR-218 via binding to its 3'UTR $(13,19)$. To investigate whether BMI-1 is involved in miR-218-induced apoptosis and growth arrest in HCC, pre-miR-218 or pre-miR-control was transfected into HepG2 and SMMC-7721 cells. As assessed by qRT-PCR and immunoblotting, miR-218 resulted in significant decrease of BMI-1 mRNA ( $<<0.05$, respectively, Fig. 5A) and protein $(\mathrm{p}<0.01$, respectively, Fig. 5B) level in both HepG2 and SMMC-7721 cells. Furthermore, we compared the expression of the BMI-1 mRNA between HCC and matched normal tumor-adjacent tissues. Our data indicated that BMI-1 mRNA level was 
A

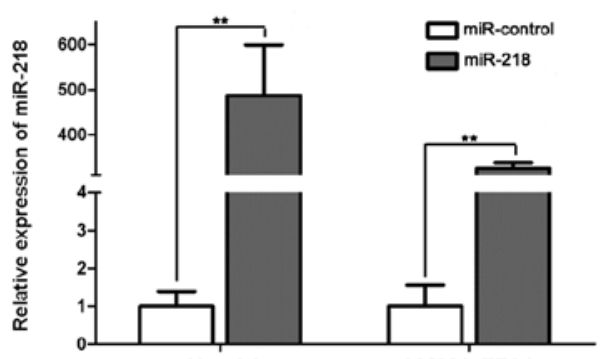

HepG2
B
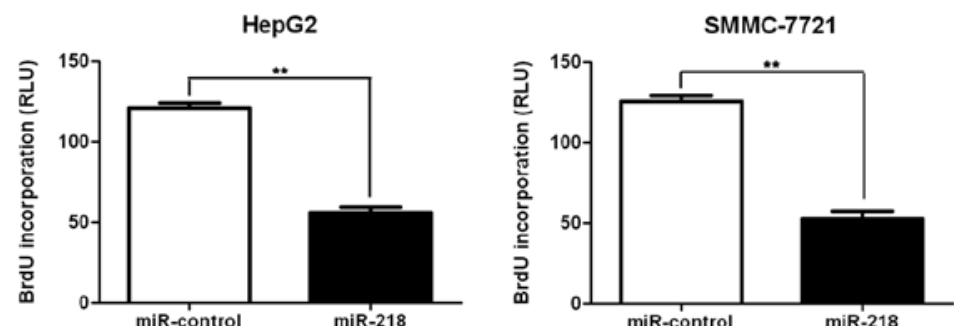

C
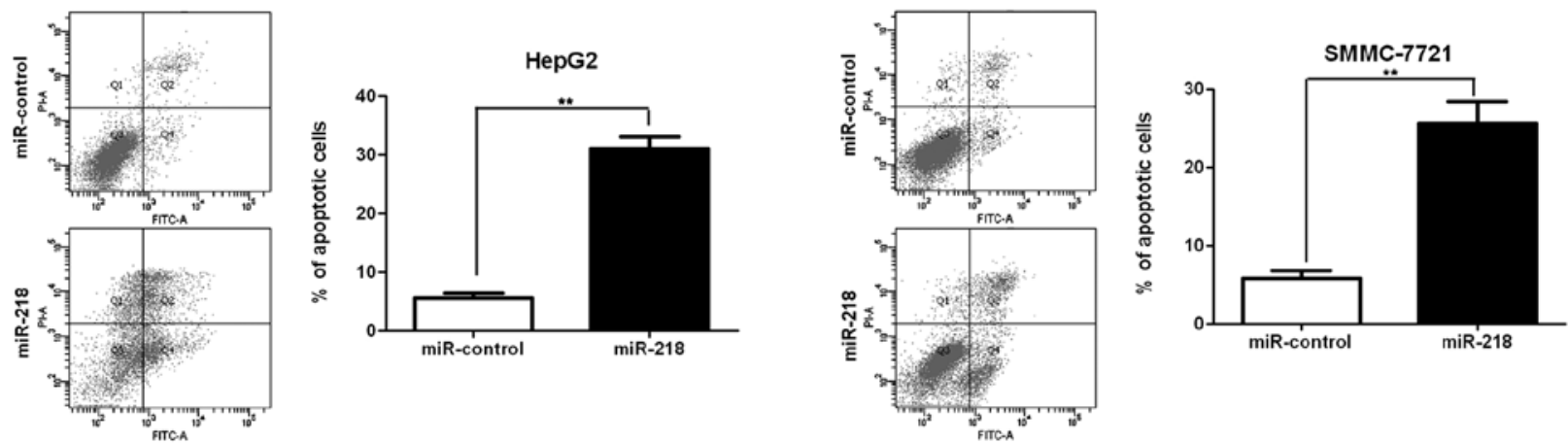

Figure 3. miR-218 regulates proliferation and apoptosis in HCC cells. (A) HepG2 and SMMC-7721 cells that were transfected with miR-control or miR-218, and subjected to qRT-PCR for miR-218. $\mathrm{n}=6$, values are depicted as means $\pm \mathrm{SEM} ;{ }^{* *} \mathrm{p}<0.01$ by $\mathrm{t}$-test. (B) Cell proliferation as assessed by BrdU cell proliferation assays was inhibited by miR-218 in HepG2 and SMMC-7721 cells. " $p<0.01$ by t-test; $n=3$ repeats with similar results. (C) Quantification of the apoptotic cell population by flow cytometry. miR-218 re-expressing HepG2 and SMMC-7721 cells were composed of a larger subset of apoptotic cells as compared with control cells. ${ }^{* * *} \mathrm{p}<0.01$ by $\mathrm{t}$-test; $\mathrm{n}=3$ repeats with similar results.

A
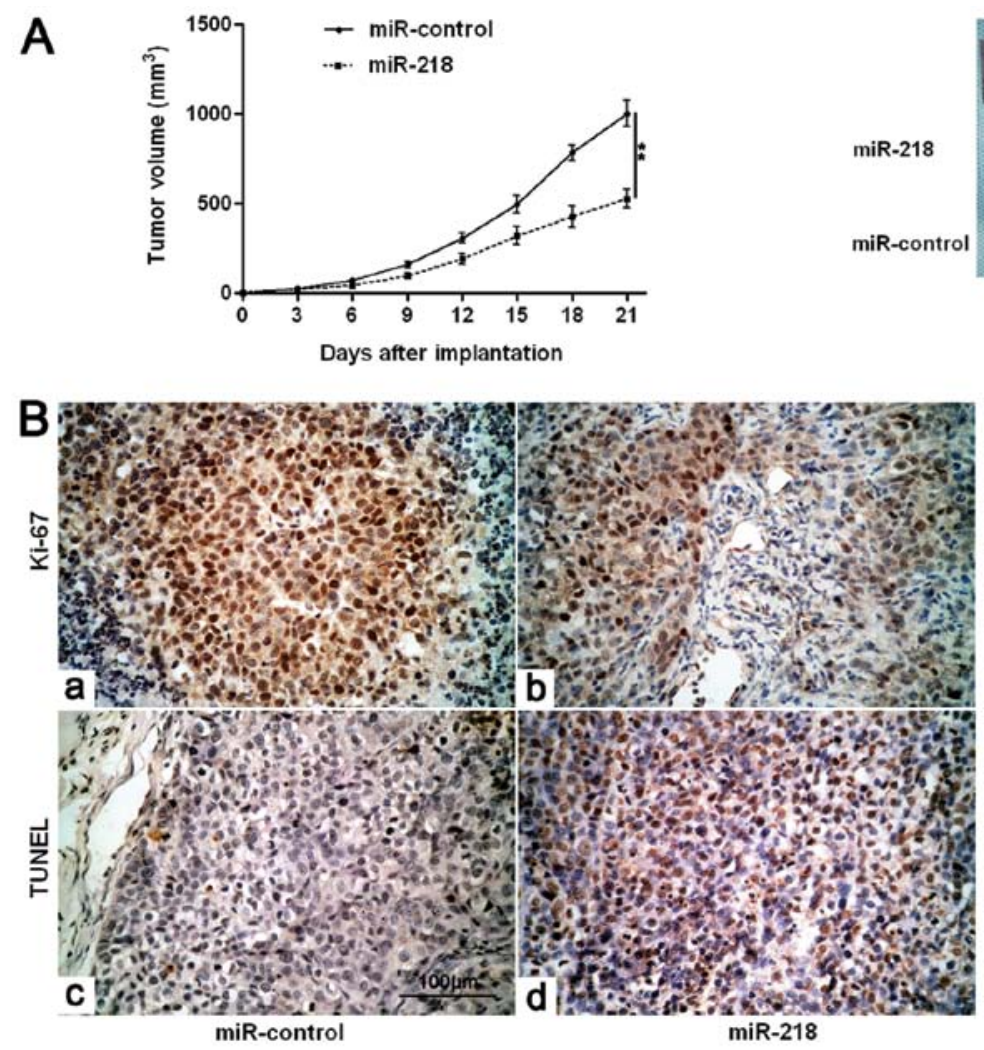
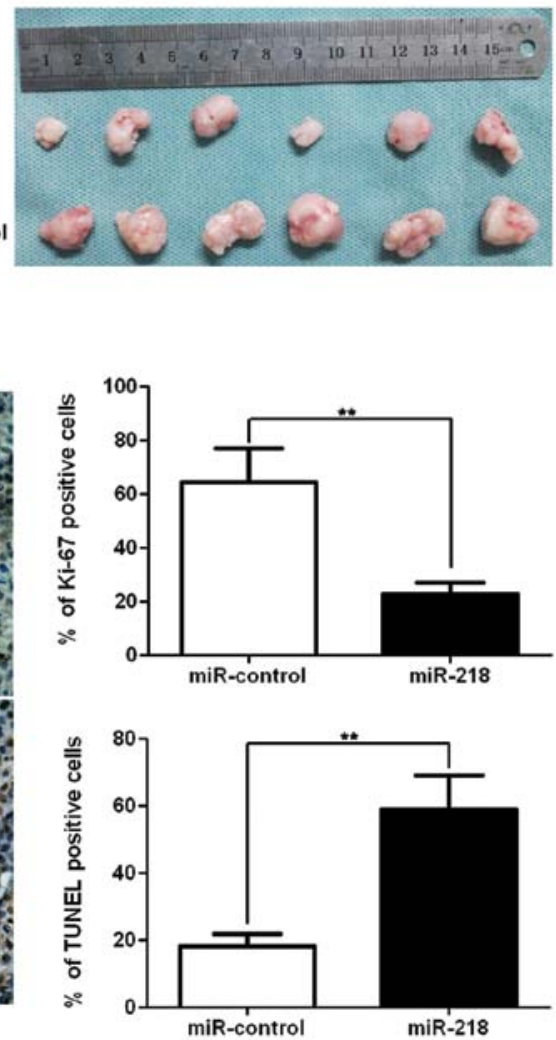

Figure 4. miR-218 suppresses tumor growth in vivo. (A) SMMC-7721 cells were implanted into nude mice via subcutaneous injection. Tumor-bearing mice were treated with miR-control $(n=6)$ or miR-218 $(n=6)$ by multicenter intratumoral injection. miR-218-treated group exhibited a higher tumor inhibiting effect compared with control group. ${ }^{* *} \mathrm{p}<0.01$ by two-way ANOVA. (B) Tumor nodules were subjected to immunohistochemical staining for Ki-67 and TUNEL assays. Representative immunostaining and TUNEL assays revealed that miR-218 significantly reduced the number of Ki-67 positive cells (a and b) and increased the number of apoptotic cells (c and d). Scale bar, $100 \mu \mathrm{m} ; \mathrm{n}=6$, values are depicted as the means $\pm \mathrm{SEM}$; ** $\mathrm{p}<0.01$ by t-test. 


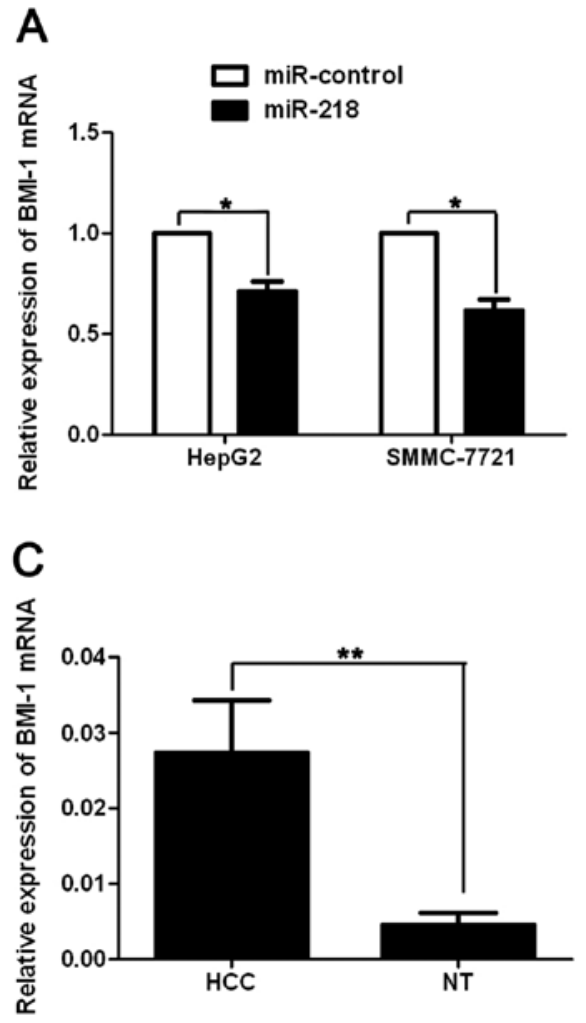

B
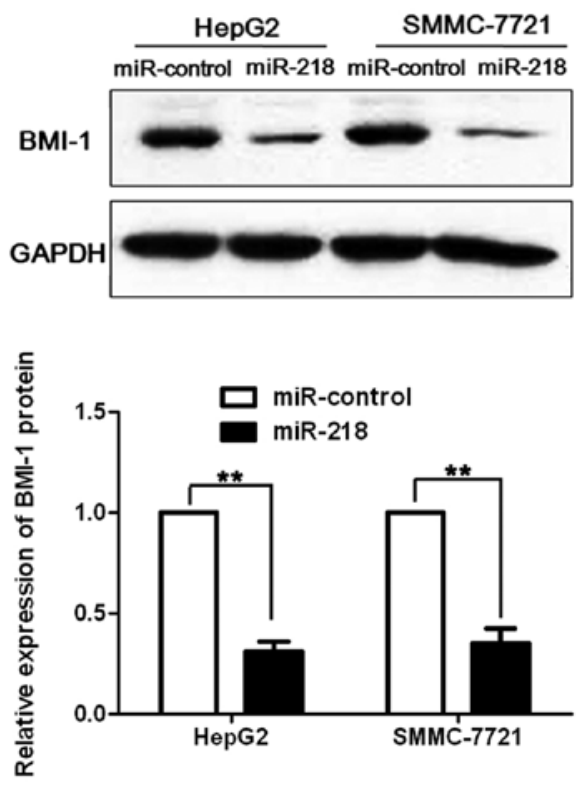

Figure 5. miR-218 regulates the expression of BMI-1 in HCC cells. (A) HepG2 and SMMC-7721 cells that were transfected with miR-control or miR-218, and subjected to qRT-PCR for BMI-1 mRNA. miR-218 decreased BMI-1 mRNA levels in HepG2 and SMMC-7721 cells. n=6, values are depicted as the means \pm SEM; " $p<0.05$ by t-test. (B) HepG2 and SMMC-7721 cells that were transfected with miR-control or miR-218, and subjected to western blotting for BMI-1 protein. miR-218 decreased BMI-1 protein levels in HepG2 and SMMC-7721 cells. ${ }^{* *} \mathrm{p}<0.01$ by t-test; $\mathrm{n}=3$ repeats with similar results. (C) The expression of BMI-1 mRNA in tumor (HCC) and non-tumor tissues (NT) from HCC patients by qRT-PCR. $\mathrm{n}=60$, values are depicted as means \pm SEM; ** $<<0.01$ by t-test.

significantly increased in $\mathrm{HCC}$ by $\sim 6$-fold compared with the non-cancerous tissues (p<0.01, Fig. 5C). Pearson's correlation analysis showed that the expression of BMI-1 was inversely correlated with miR-218 in HCC tissues $(r=-0.572, p=0.003)$. Taken together, these data indicate that BMI-1 may function as a downstream factor in miR-218-induced apoptosis and growth arrest in HCC.

\section{Discussion}

HCC is the most common primary tumor in liver and the third most frequent malignant tumor due to the high incidence of HBV infection in China. Several studies reported that miRNAs regulate carcinogenesis-related gene expression, suggesting a new mechanism involved in HCC initiation and development. We primarily detected miR-218 expression in 60 samples of paired HCC and normal tumor-adjacent tissues using qRT-PCR; our data indicated that miR-218 level in the cancer tissues was significantly lower than that in the noncancerous tissues. Furthermore, miR-218 was expressed at significantly lower levels in HCC patients with large tumor size, high Edmondson-Steiner grading and advanced TNM tumor stage. These results are consistent with the status and clinical significance of miR-218 in other types of human cancer including colorectal and pancreatic cancer, and glioma $(13,20,21)$. Notably, our data showed that reduced miR-218 expression conferred a significantly poorer 5 -year patient survival for HCC patients. Multivariate Cox regression analysis indicated that miR-218 was an independent factor for predicting both overall 5-year survival and disease-free survival in HCC patients. Collectively, these results show that the status of miR-218 is critical for prognosis determination in HCC patients.

Functional studies demonstrated that miR-218 suppresses proliferation, inhibits cell cycle progression and induces apoptosis in colorectal cancer and glioma $(20,21)$. Furthermore, miR-218 suppresses cell migration and invasion in gastric cancer, cervical squamous cell carcinoma, lung cancer and thyroid cancer and glioma $(11,21)$. In the present study, we showed that restoring miR-218 expression led to reduced cell proliferation and elevated apoptotic HCC cells. Ectopically expressing miR-218 conferred an inhibitory effect on tumor growth in a nude mouse xenograft model. Furthermore, immunostaining of Ki-67 and TUNEL assays indicated miR-218 may suppress tumor growth by inducing apoptosis and growth arrest in vivo.

B lymphoma Mo-MLV insertion region 1 homolog (BMI-1), a member of the polycomb group (PcG), functions as a transcriptional repressor and presents with high expression in many tumors including $\mathrm{HCC}$, indicating a poor prognosis $(22,23)$. BMI-1 has been shown to be an oncogene that regulates cell proliferation and transformation (19). BMI-1 is also critical for the self-renewal of stem cells and cancer initiation $(24,25)$. Several recent studies reported that miR-218 inhibited 
tumor progression by targeting the polycomb group gene Bmi-1 (13,21). miR-218 could directly bind to 3'-UTR of Bmi-1 and subsequently suppress BMI-1 protein expression $(13,21)$. We investigated the regulatory effect of miR-218 on BMI-1 in HCC and our data showed that ectopically expressing miR-218 resulted in evident reduction of both BMI-1 mRNA and protein level in two different HCC cell lines. We found that BMI-1 mRNA was significantly higher in HCC tissues than in matched normal tumor-adjacent tissues, which has been reported in previous studies (26). Furthermore, Pearson's correlation analysis indicated that miR-218 expression was negatively correlated with BMI-1 mRNA expression in HCC tissues. Thus, BMI-1 may be a downstream target of miR-218 in HCC.

In conclusion, we demonstrated that miR-218 expression is impaired in HCC and reduced levels of miR-218 are associated with poor clinicopathological characteristics. HCC patients with low expression of miR-218 exhibit a poor 5-year survival. miR-218 is an independent factor for predicting poor prognosis in HCC patients. miR-218 acts as an HCC tumor suppressor by inhibiting cell proliferation and promoting apoptosis in vitro and in vivo. BMI-1 may be a target for miR-218 and its abundance is inversely regulated by miR-218 in HCC cells. Collectively, we hypothesize that loss of miR-218 function contributes to hepatocarcinogenesis, in part through the accumulation of BMI-1. We identified miR-218 as a potential therapeutic target for $\mathrm{HCC}$.

\section{Acknowledgements}

This study was supported by a grant from the National Natural Science Foundation of China (nos. 81272645 and 81071897).

\section{References}

1. Tu K, Zheng X, Zan X, Han S, Yao Y and Liu Q: Evaluation of Fbxw7 expression and its correlation with the expression of c-Myc, cyclin E and p53 in human hepatocellular carcinoma. Hepatol Res 42: 904-910, 2012.

2. Tu K, Zheng X, Zhou Z, et al: Recombinant human adenovirusp53 injection induced apoptosis in hepatocellular carcinoma cell lines mediated by p53-Fbxw7 pathway, which controls c-Myc and cyclin E. PLoS One 8: e68574, 2013.

3. Ambros V: The functions of animal microRNAs. Nature 431: 350-355, 2004.

4. Jia Z, Wang K, Wang G, Zhang A and Pu P: MiR-30a-5p antisense oligonucleotide suppresses glioma cell growth by targeting SEPT7. PLoS One 8: e55008, 2013.

5. Baer C, Claus R and Plass C: Genome-wide epigenetic regulation of miRNAs in cancer. Cancer Res 73: 473-477, 2013.

6. Wong CM, Kai AK, Tsang FH and Ng IO: Regulation of hepatocarcinogenesis by microRNAs. Front Biosci 5: 49-60, 2013.

7. Gramantieri L, Fornari F, Callegari E, et al: MicroRNA involvement in hepatocellular carcinoma. J Cell Mol Med 12: 2189-2204, 2008.
8. Davidson MR, Larsen JE, Yang IA, et al: MicroRNA-218 is deleted and downregulated in lung squamous cell carcinoma. PLoS One 5: e12560, 2010.

9. Chiyomaru T, Enokida H, Kawakami K, et al: Functional role of LASP1 in cell viability and its regulation by microRNAs in bladder cancer. Urol Oncol 30: 434-443, 2012.

10. Song L, Huang Q, Chen K, et al: miR-218 inhibits the invasive ability of glioma cells by direct downregulation of IKK- $\beta$. Biochem Biophys Res Commun 402: 135-140, 2010.

11. Tie J, Pan Y, Zhao L, et al: MiR-218 inhibits invasion and metastasis of gastric cancer by targeting the Robol receptor. PLoS Genet 6: e1000879, 2010.

12. Li J, Ping Z and Ning H: MiR-218 impairs tumor growth and increases chemo-sensitivity to cisplatin in cervical cancer. Int J Mol Sci 13: 16053-16064, 2012.

13. He X, Dong Y, Wu CW, et al: MicroRNA-218 inhibits cell cycle progression and promotes apoptosis in colon cancer by downregulating BMI1 polycomb ring finger oncogene. Mol Med 18: 1491-1498, 2012.

14. Leite KR, Sousa-Canavez JM, Reis ST, et al: Change in expression of miR-let7c, miR-100, and miR-218 from high grade localized prostate cancer to metastasis. Urol Oncol 29: 265-269, 2011.

15. Uesugi A, Kozaki K, Tsuruta T, et al: The tumor suppressive microRNA miR-218 targets the mTOR component Rictor and inhibits AKT phosphorylation in oral cancer. Cancer Res 71: 5765-5778, 2011

16. Li BS, Zhao YL, Guo G, et al: Plasma microRNAs, miR-223, miR-21 and miR-218, as novel potential biomarkers for gastric cancer detection. PLoS One 7: e41629, 2012.

17. Zheng X, Gai X, Ding F, Lu Z, Tu K, Yao Y and Liu Q: Histone acetyltransferase PCAF up-regulated cell apoptosis in hepatocellular carcinoma via acetylating histone $\mathrm{H} 4$ and inactivating AKT signaling. Mol Cancer 12: 96, 2013.

18. Tu K, Zheng X, Yin G, Zan X, Yao Y and Liu Q: Evaluation of Fbxw7 expression and its correlation with expression of SREBP-1 in a mouse model of NAFLD. Mol Med Rep 6: 525-530, 2012.

19. Kang MK, Kim RH, Kim SJ, et al: Elevated Bmi-1 expression is associated with dysplastic cell transformation during oral carcinogenesis and is required for cancer cell replication and survival. Br J Cancer 96: 126-133, 2007.

20. Zhu Z, Xu Y, Du J, Tan J and Jiao H: Expression of microRNA-218 in human pancreatic ductal adenocarcinoma and its correlation with tumor progression and patient survival. J Surg Oncol 109: 89-94, 2014.

21. Tu Y, Gao X, Li G, et al: MicroRNA-218 inhibits glioma invasion, migration, proliferation, and cancer stem-like cell self-renewal by targeting the polycomb group gene Bmil. Cancer Res 73: 6046-6055, 2013

22. Tong YQ, Liu B, Zheng HY, He YJ, Gu J, Li F and Li Y: Overexpression of BMI-1 is associated with poor prognosis in cervical cancer. Asia Pac J Clin Oncol 8: e55-e62, 2012.

23. Yin $\mathrm{T}$, Wei $\mathrm{H}$, Leng $\mathrm{Z}$, et al: Bmi-1 promotes the chemoresistance, invasion and tumorigenesis of pancreatic cancer cells. Chemotherapy 57: 488-496, 2011.

24. Schuringa JJ and Vellenga E: Role of the polycomb group gene BMI1 in normal and leukemic hematopoietic stem and progenitor cells. Curr Opin Hematol 17: 294-299, 2010.

25. Douglas D, Hsu JH, Hung L, et al: BMI-1 promotes ewing sarcoma tumorigenicity independent of $C D K N 2 A$ repression. Cancer Res 68: 6507-6515, 2008.

26. Li X, Yang Z, Song W, et al: Overexpression of Bmi-1 contributes to the invasion and metastasis of hepatocellular carcinoma by increasing the expression of matrix metalloproteinase (MMP)-2, MMP-9 and vascular endothelial growth factor via the PTEN/ PI3K/Akt pathway. Int J Oncol 43: 793-802, 2013. 